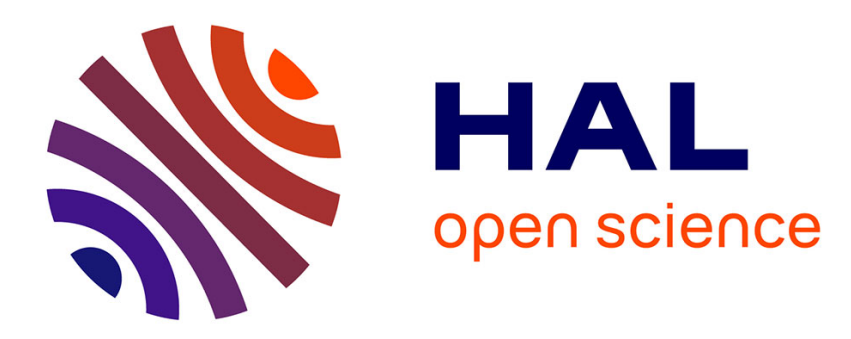

\title{
Un bloc architectural retaillé en sarcophage au musée d'Arles.
}

\author{
Vassiliki Gaggadis-Robin
}

\section{To cite this version:}

Vassiliki Gaggadis-Robin. Un bloc architectural retaillé en sarcophage au musée d'Arles.. Revue archéologique, 2014, 2014, 2. hal-01461405

\section{HAL Id: hal-01461405 \\ https://hal.science/hal-01461405}

Submitted on 8 Feb 2017

HAL is a multi-disciplinary open access archive for the deposit and dissemination of scientific research documents, whether they are published or not. The documents may come from teaching and research institutions in France or abroad, or from public or private research centers.
L'archive ouverte pluridisciplinaire HAL, est destinée au dépôt et à la diffusion de documents scientifiques de niveau recherche, publiés ou non, émanant des établissements d'enseignement et de recherche français ou étrangers, des laboratoires publics ou privés. 


\section{REVUE ARCHÉOLOGIQUE}

\section{4 - Fascicule 2}

\section{Sommaire}

La redécouverte d'un buste antique sicilien en terre cuite de la collection Janzé, par Cécile Colonna et Nathalie Buisson

Journée d'hommage à Jean Marcadé, 24 janvier 2014, Paris, Institut national d'histoire de l'art et Institut de France

Avant-propos, par Marie-Christine Hellmann

Apollon et le Colosse des Naxiens, par François Queyrel

Jean Marcadé et Praxitèle à Délos, par Francis Prost

Une petite énigme délienne : les « curieuses offrandes » des hiéronautes de Tyr, par Antoine Hermary

Un Apollon remarquable : analogies thématiques dans la sculpture hellénistique de Rhodes et de Délos, par Vasiliki Machaira

La Victoire de Samothrace en restauration : gros plan sur la cuvette d'encastrement, par Marianne Hamiaux

Une figure recomposée de la frise de l'Érechthéion, par Raphaël Jacob

Un relief exceptionnel de Thasos, par Bernard Holtzmann

Un bloc architectural retaillé en sarcophage au musée d'Arles, par Vassiliki Gaggadis-Robin

Une nouvelle stèle funéraire attique, dédiée à la mémoire de Jean Marcadé,

par Georges Despinis $\uparrow$

Les couleurs et les ors retrouvés de la sculpture antique..., par Philippe Jockey

Des mots pour les choses, selon Jean Marcadé, conclusion de la journée d'hommage

à Jean Marcadé, par Alain Pasquier

Comptes rendus bibliographiques

Ouvrages adressés à la Revue archéologique

Table annuelle

ISBN : 978-2-13-062916-0 


\title{
UN BLOC ARCHITECTURAL RETAILLÉ EN SARCOPHAGE AU MUSÉE D'ARLES
}

\author{
par Vassiliki Gaggadis-Robin
}

\begin{abstract}
Résumé. - Cet article traite du sarcophage paléochrétien dit "de l'Orante ", conservé au Musée départemental Arles antique. Il est taillé dans un bloc architectural en marbre de Carrare qui a gardé les traces de son décor d'origine : des Nymphes ou des Charites qui se dirigent vers un thymiaterion. Le sujet figuré, le traitement de ce qui reste des draperies, le type du brûle-parfum situent ce bloc parmi les réalisations néo-attiques d'époque augustéenne. Pour tailler la cuve, la frise a été arasée dans la seconde moitié du IV e. Par la comparaison avec des cuves de Rome, l'auteur pense que ce bloc ornait à l'origine un monument païen, peut-être à Rome; après son démantèlement, le bloc a été retaillé par des ateliers sculptant des sarcophages chrétiens à Rome et importé par la suite à Arles.
\end{abstract}

Mots clés. - Rome. Arles/Arelatum. Sculpture. Sarcophage. Marbre. Remploi. Nymphes. Orante. Apôtre. Époque augustéenne. Époque paléochrétienne.
An architectural block re-cut as sarcophagus in the Museum of Ancient Arles

Abstract. - This article is about the early Christian sarcophagus called "the praying lady" in the Musée départemental Arles antique. It is carved from an architectural block of Carrara marble, which has preserved traces of its original decoration: Nymphs or Charites walking to a thymiaterion. The representation and the treatment of the remaining drapery, with the type of incense burner, date this block with neo-Attic production of the Augustan period. In the second half of the 4 th cent. AD the frieze was levelled for carving the sarcophagus. Comparing chests in Rome, the author believes that this block originally adorned a pagan building, perhaps in Rome, which had been dismantled, the stone then being re-cut by workshops carving Christian sarcophagi in Rome and taken later to Arles.

Key-Words. - Rome. Arles/Arelatum. Sculpture. Sarcophagus. Marble. Re-use. Nymphs. Praying lady. Apostle. Augustan period. Early Christian period.

La pièce dont il sera question ici, telle qu'on peut la voir au musée d'Arles, est un sarcophage à décor paléochrétien. Ce sujet pourrait sembler assez éloigné de la spécialité de Jean Marcadé, tant par son domaine typologique que par son iconographie, mais d'une part notre Maître n'a jamais refusé d'élargir son champ d'étude, et, d'autre part, ce sarcophage se rattache bien à deux de ses centres d'intérêt permanents : les reliefs néo-attiques ou archaïsants et les techniques de mise en œuvre des sculptures, si bien que la discussion que nous avons eue, au moment de la découverte des particularités de ce bloc, m'a été d'un grand secours.

Le sarcophage dit "de l'Orante "1, retrouvé aux Alyscamps vers 1847 (fig. 1), a pour dimensions $0,54 \mathrm{~m}$ de hauteur, $2,22 \mathrm{~m}$ de longueur et $0,68 \mathrm{~m}$ de largeur. Il y subsiste de la dorure et il est taillé dans un bloc architectural dont quelques traces du décor originel (fig. 2) sont conservées.

1. Musée départemental Arles antique: Inv. FAN 92. 00. 2512 : BeNorT 1954, p. $60 \mathrm{n}^{\circ} 76$, pl. XXXI, 2 et 6 ;

Rev. ARCH. 2/2014, p. 335-346.
Christern-Briesenick 2003, p. 56 n 75, pl. 27, 2 ; GagGadisRobin, Maniatis, Polykreti 2009. 


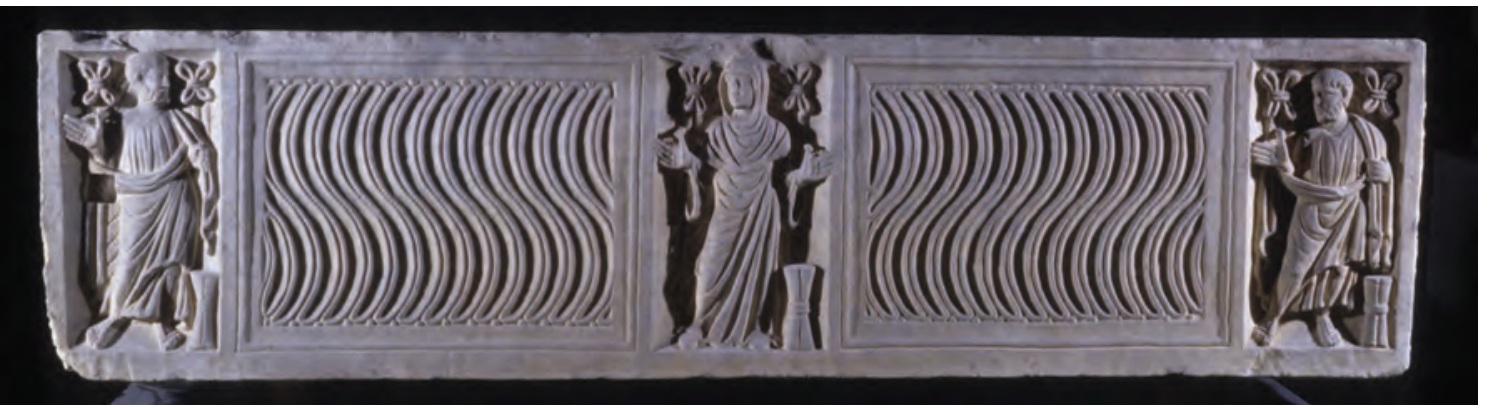

1. Sarcophage dit " de l'Orante ", face. Musée départemental Arles antique (MDAA). (C) Cl. Ch. Durand, G. Réveillac, CNRS, Centre Camille-Jullian (CCJ).

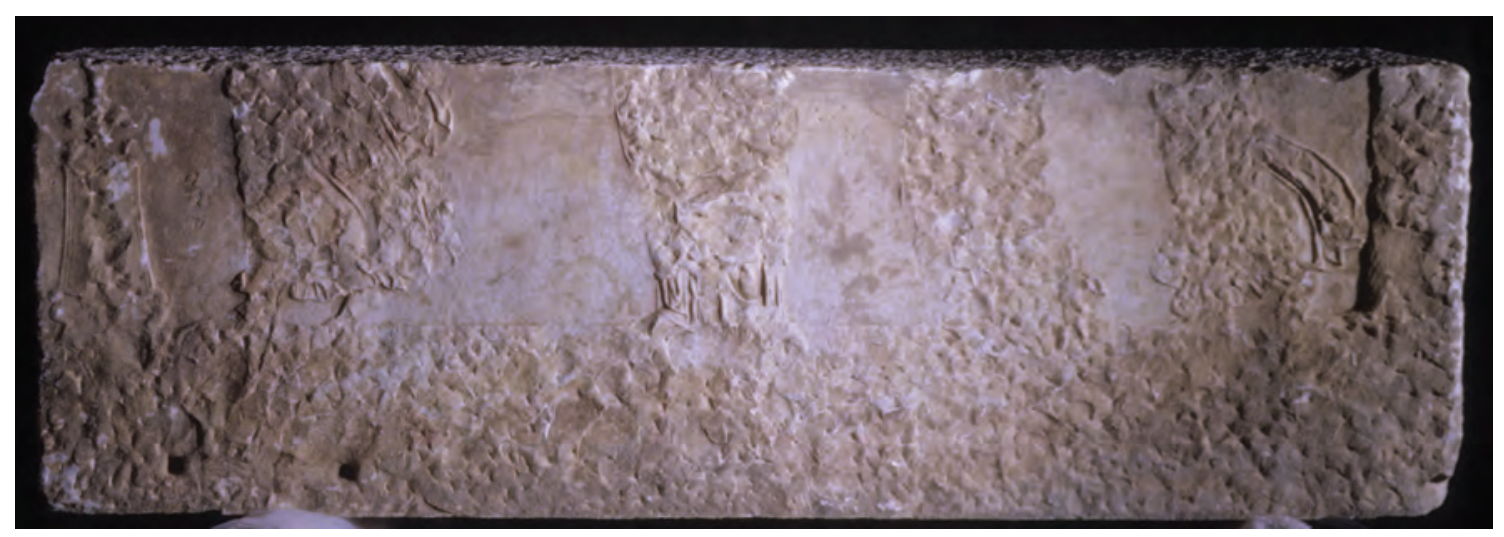

2. Face extérieure du fond du sarcophage dit « de l'Orante ». MDAA.

(c) Cl. Ch. Durand, G. Réveillac, CNRS, CCJ.

Le matériau est du marbre de Carrare. Des échantillons de marbre prélevés ont été analysés par Kyriaki Polykreti et Yannis Maniatis du laboratoire Dimocritos à Athènes, selon la technique de spectroscopie par résonance paramagnétique EPR (Electron Paramagnetic Resonance) ${ }^{2}$. Avant l'analyse, les échantillons ont été examinés au microscope puis au stéréoscope, pour déterminer la couleur du marbre et évaluer la taille maximale des grains des cristaux (Maximum Grain Size MMK). Comparés à la base de données du laboratoire, qui contient des échantillons de toutes les carrières de marbre antiques connues, les résultats obtenus ont montré qu'il s'agissait de marbre de Carrare. 


\section{LE DÉCOR DU SARCOPHAGE ET SA DATATION}

Le décor chrétien du sarcophage est assez fréquent (fig. 1). La face est partagée en cinq panneaux parmi lesquels les deux plus longs sont ornés de strigiles, motifs décoratifs souvent employés sur les sarcophages, de diverses façons, dès la fin du II $^{\mathrm{e}} \mathrm{s}^{3}$ Dans les trois panneaux figurés, dont les angles supérieurs sont occupés par une draperie nouée de manière stylisée et décorative, se détachent des personnages. Au centre une figure d'Orante (fig. 3), probablement la défunte, voilée et vue de face, la tête tournée vers la gauche; elle est vêtue d'une longue tunique à manches larges et d'un châle court qui lui couvre la tête, ses deux bras sont levés en posture de prière. Le visage ovale est fin, le nez long, la bouche petite aux lèvres charnues, les yeux en amande ont la pupille creusée d'un coup de foret, une ride marque le front. Dans chaque panneau des extrémités un apôtre se tient de face, faisant le geste de l'acclamation (fig. 4-5), la tête tournée vers le centre, vers l'Orante. Chacun est vêtu d'une tunique, d'un manteau et de sandales. Leur visage fin a les mêmes caractéristiques que celui de l'Orante, les cheveux sont coiffés vers l'avant, les boucles de la barbe courte marquées par les stries. Aux pieds des trois personnages se trouve un faisceau de rouleaux. Les strigiles (fig. 6) sont élégamment ornés à leurs extrémités de petites perles, un détail qui se retrouve surtout sur les exemplaires les plus soignés de cette série ${ }^{4}$. L'iconographie du personnage en prière, accompagné par des apôtres, apparaît d'abord sur les sarcophages à frise continue dans le premier quart du $\mathrm{IV}^{\mathrm{e}} \mathrm{s}$. avant d'être reprise par la suite sur les cuves à panneaux.

Le décor figuré date le remploi du bloc en sarcophage et l'exécution des panneaux de la deuxième moitié $d u \mathrm{IV}^{\mathrm{e}} \mathrm{s}$. Le motif des strigiles a été amplement utilisé pour le décor des cuves au cours $\mathrm{du} I \mathrm{II}^{\mathrm{e}} \mathrm{s}$. et une partie du IV $\mathrm{v}^{\mathrm{e}}$, mais les figures d'Orant et des apôtres datent du IV $\mathrm{V}^{\mathrm{e}} \mathrm{s}$.

\section{LE DÉCOR DU BLOC ARCHITECTURAL ET SA DATATION}

Cette pièce, apparemment assez commune, gagne en intérêt si l'on y reconnaît le remploi d'un bloc architectural, ce qui a été remarqué lors du déménagement des pièces dans le nouveau musée, inauguré en 1996.

Alors que les deux petits côtés et l'arrière du sarcophage chrétien (fig. 7-8) sont travaillés à la pointe, la face extérieure du fond de la cuve a gardé les traces du décor que portait le bloc lors de son premier emploi (fig. 2). Le sculpteur du sarcophage a pris soin d'araser au ciseau et à la pointe toute la partie qui porte le décor originel du bloc, mais des éléments en très bas relief sont encore visibles. On peut ainsi reconnaître quatre figures humaines sur un fond qui est délimité à droite et dans sa partie inférieure par une moulure assez large. Peut-être qu'à gauche également une autre moulure encadrait le relief. Un objet de grande taille se devine à gauche (fig. 9), une base dont les

3. G. Koch, H. SichtermanN, Römische Sarkophage, Munich, Beck, 1982, p. 73-76.
4. Parmi de nombreux ex. citons Apt : CHRISTERn-Briesenick 2003, p. 14-15 n $\mathrm{n}^{\circ}$ 30-31, pl. 9-10; Arles : CHRISTERNBRIESENICK 2003, p. 57 n 77 , pl. 28 ; p. 59 n 81 , pl. 29. 


\section{- (c) PUF}

3 novembre 2014 11:26 - Revue archéologique_2/2014 - AUTEUR - Revue archéologique - 217 x 270 - page 338 / 448

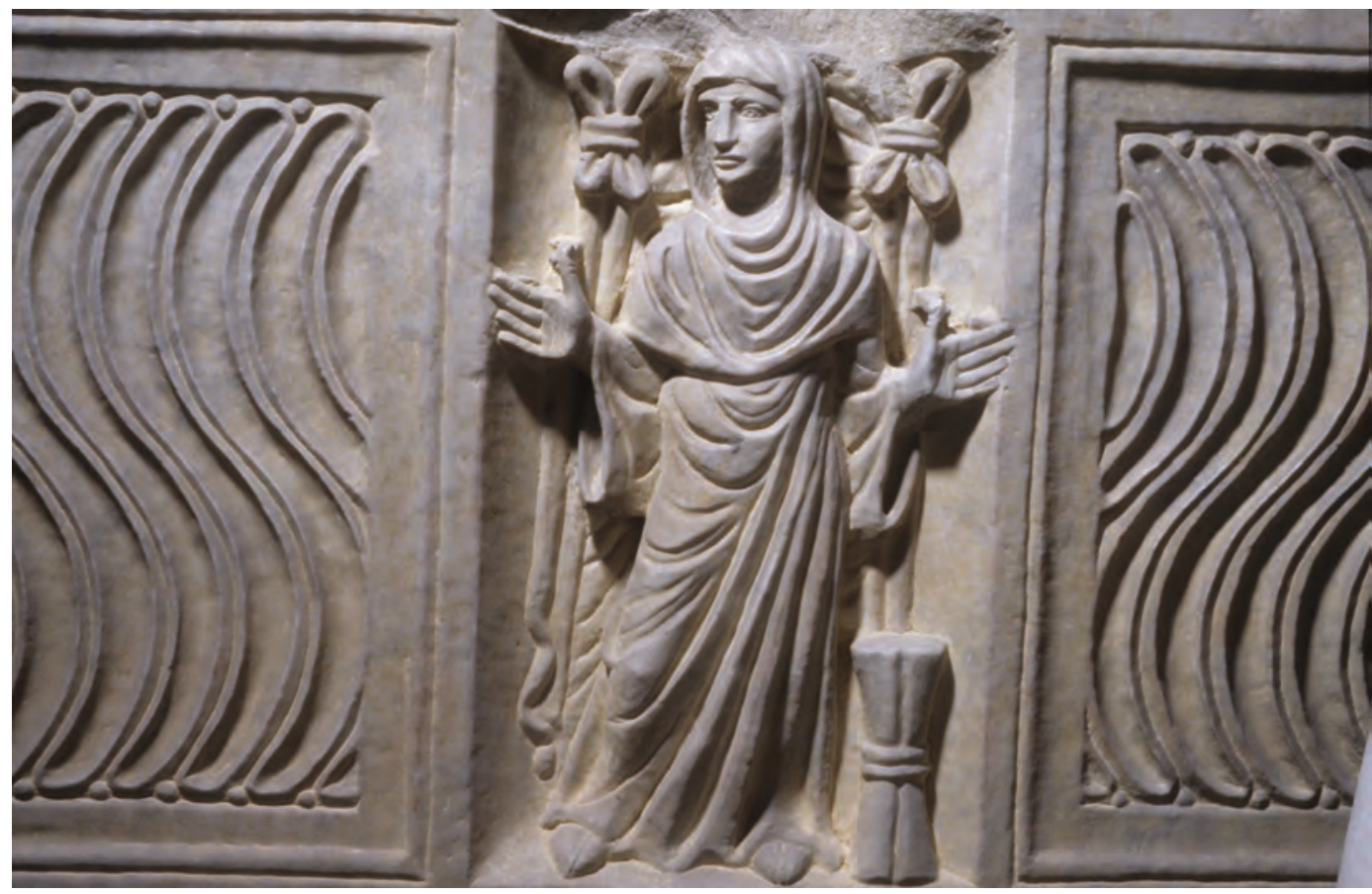

3. Sarcophage dit « de I'Orante ", panneau central. MDAA. (c) Cl. Ch. Durand, G. Réveillac, CNRS, CCJ.

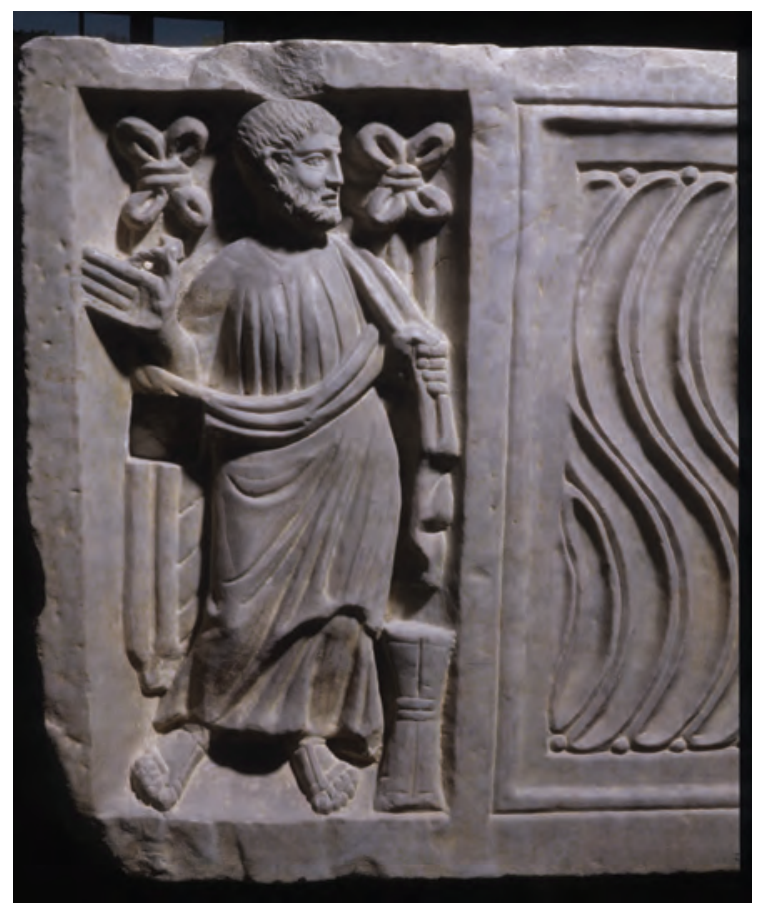

4. Sarcophage dit "de l'Orante ", panneau de gauche. MDAA. (c) Cl. Ch. Durand, G. Réveillac, CNRS, CCJ. 
5. Sarcophage dit " de l'Orante ", panneau de droite. MDAA. (C) Cl. Ch. Durand, G. Réveillac, CNRS, CCJ.
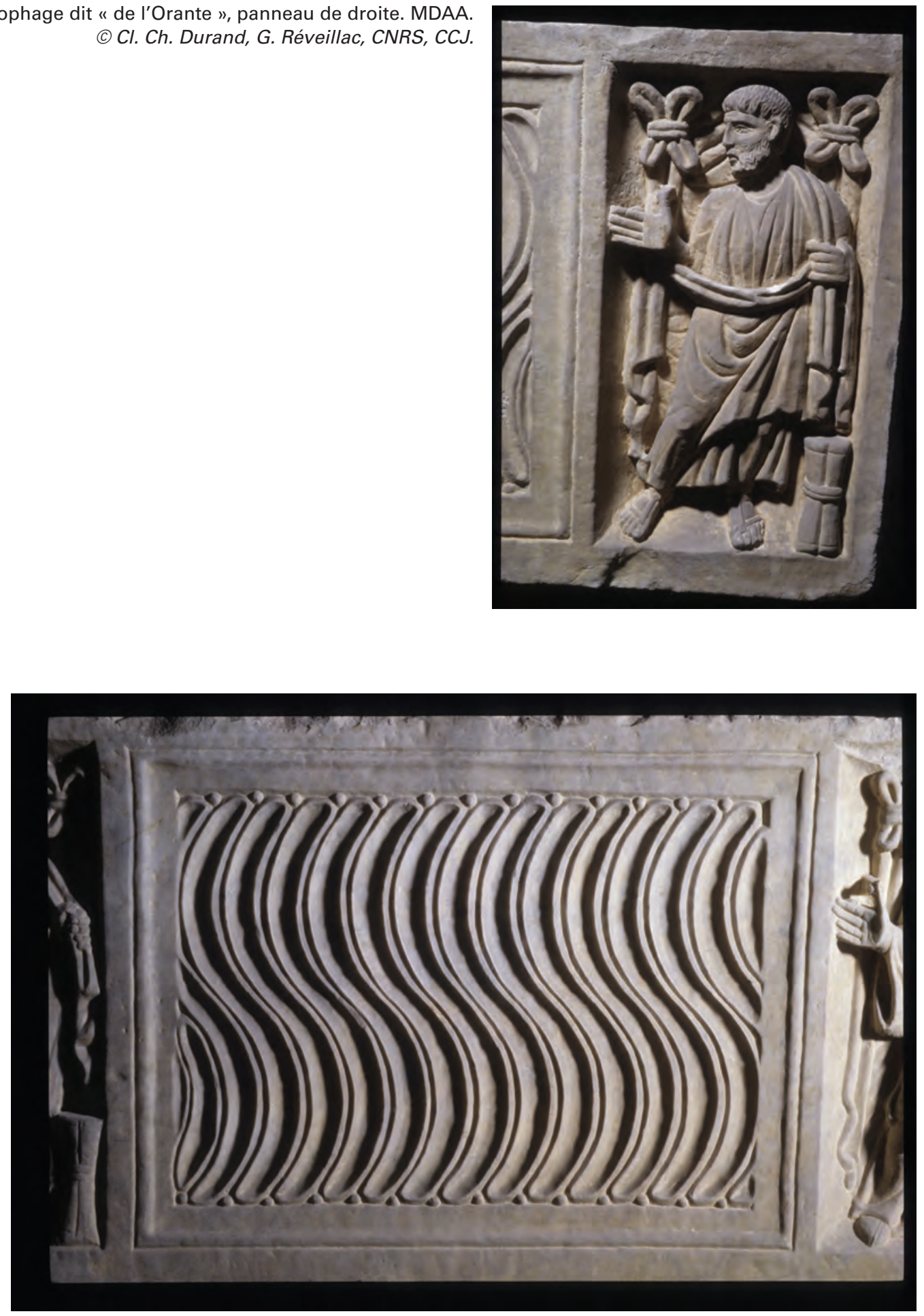

6. Sarcophage dit " de l'Orante ", panneau des strigiles. MDAA. ( C Cl. Ch. Durand, G. Réveillac, CNRS, CCJ. 


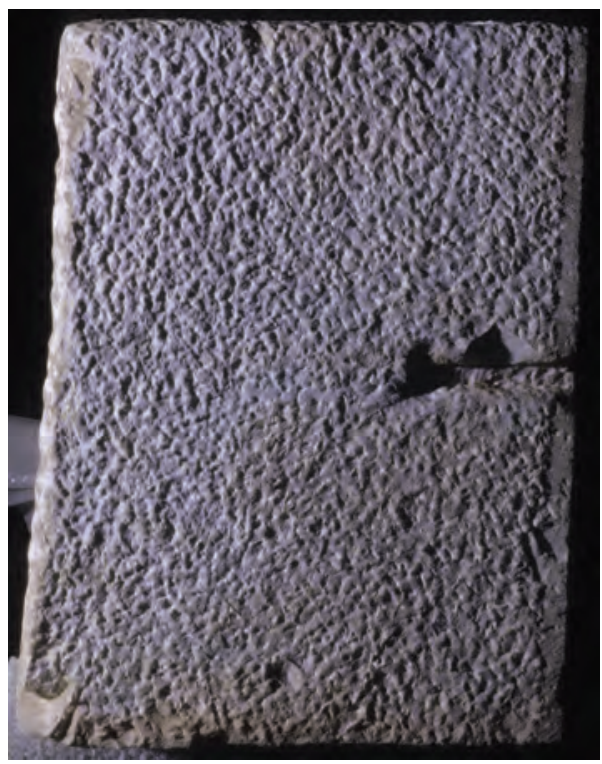

7. Sarcophage dit " de l'Orante ", petit côté gauche. MDAA.

(c) Cl. Ch. Durand, G. Réveillac, CNRS, CCJ.

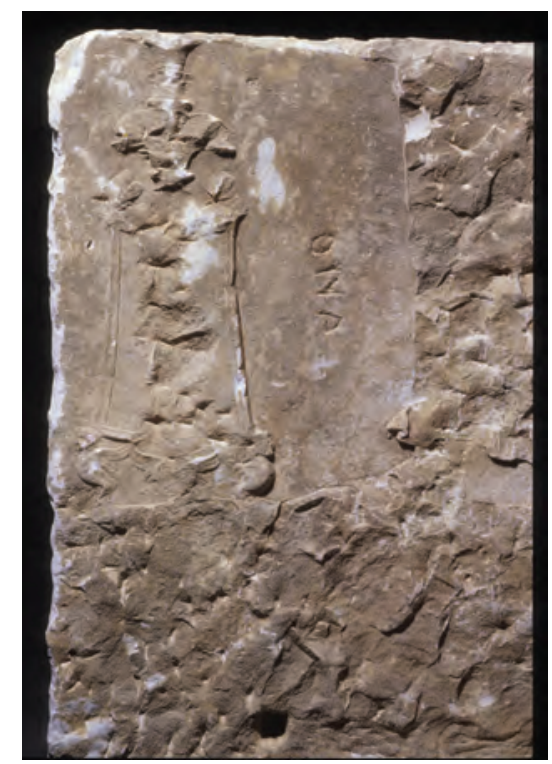

8. Fond du sarcophage dit " de l'Orante", détail de la partie gauche. MDAA. (C) Cl. Ch. Durand, G. Réveillac, CNRS, CCJ.

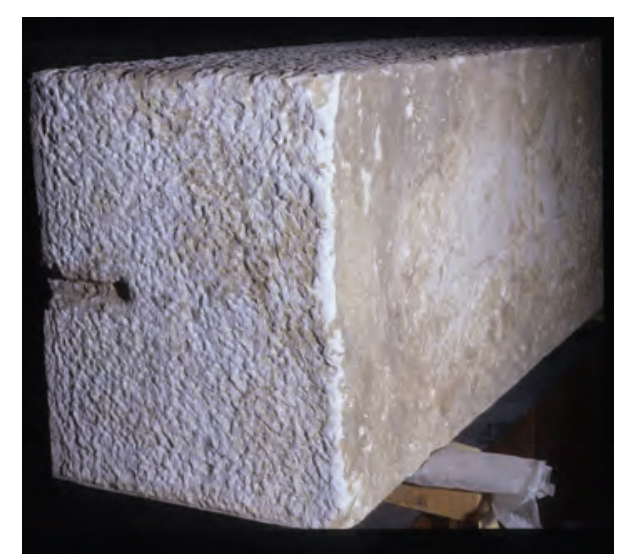

9. Face extérieure du fond du sarcophage, détail de la partie gauche. MDAA. (C) Cl. Ch. Durand, G. Réveillac, CNRS, CCJ.

pieds prennent la forme de sphinges ou de sirènes, et qui supportait certainement un thymiaterion, ou un turibulum. Dans le champ à droite de l'objet se distinguent trois lettres d'un graffito sur lequel je reviendrai plus loin. Les figures féminines (fig. 2), conservées presque à mi-hauteur, portent de longues tuniques tombant jusqu'au sol. La première figure de gauche pose à peine ses pieds sur 
le sol, dans un mouvement rapide et gracieux. La deuxième semble, par la position des pieds, être présentée de face, mais les autres paraissent se diriger de profil vers la gauche, donc vers le trépied.

Pour tailler la cuve le bloc architectural a été retourné, sa face ornée devenant le fond de la cuve, et la frise a été arasée. Il a été évidé et l'on a procédé à la taille du décor organisé en panneaux alternés sur l'ancien lit d'attente du bloc, qui est devenu ainsi la face principale du sarcophage. Dans le panneau de gauche, sous la main droite de l'apôtre, une cavité assez importante, probablement aménagée pour une pince de levage du bloc, a peut-être été soigneusement bouchée à un moment avec du stuc, ce que suggère la photographie publiée en 1954 dans l'ouvrage de Benoit ${ }^{5}$. Mais nous ne sommes pas en mesure de préciser si cette modification a été effectuée au moment de la taille du sarcophage ou au XIx ${ }^{\mathrm{e}}$ s., lors de sa découverte. À la suite des nettoyages minutieux auxquels il a été procédé en vue de l'installation des sarcophages dans le nouveau musée, la pièce a perdu ce " rajout».

Ce trou de pince indique que la face ornée du sarcophage était au départ le lit d'attente du bloc. Notons également que ce fait a entraîné un renversement des dimensions : si la longueur du bloc est restée identique (peut-être a-t-il tout de même été retaillé à gauche ?), sa hauteur d'origine, $68 \mathrm{~cm}$, est devenue la largeur du sarcophage et sa largeur d'origine, $54 \mathrm{~cm}$, est devenue la hauteur de la cuve. Le trou de pince indique également que la frise sculptée lors du premier emploi du bloc se développait au moins sur deux assises et, par conséquent, sur plus d'un mètre de hauteur.

La base du brûle-parfum, à gauche, représente un de ces objets de luxe, de grande taille et en marbre, étudiés par Hans-Ulrich Cain ${ }^{6}$. D'après ce que l'on peut distinguer sur le relief, l'objet pourrait correspondre au premier type (fig. 10) dans la typologie établie par cet auteur ${ }^{7}$. On devine sa constitution en trois parties : la base de forme tronconique à trois faces encadrées par des moulures ; les trois pieds en forme d'animal, sphinges ou sirènes sur notre exemplaire, d'après le dessin général ; la première partie de la tige enveloppée par des feuilles d'acanthe superposées qui supportaient le brûle-parfum. Notre exemplaire semble assez chargé puisqu'une tête de Méduse est sculptée entre les monstres et une des faces de la base était peut-être ornée d'une figure, arasée également lors de la retaille du bloc ${ }^{8}$. Ce type existe depuis le dernier quart du $\mathrm{II}^{\mathrm{e}} \mathrm{s}$. av. J.-C., mais la plupart des exemplaires étudiés par Cain datent de la période augustéenne.

Ce qui subsiste d'un graffito (fig. 8), entre le brûle-parfum et la première figure, mérite qu'on s'y attarde. Les trois lettres pourraient être lues hâtivement ONA. Mais il résulte d'une lecture plus attentive que la première lettre pourrait être en fait un L et un $\mathrm{O}$ ligaturés ${ }^{9}$, et la deuxième lettre pourrait être le chiffre II, puisque ce qui devait être la barre diagonale de la lettre N n'est pas gravé de manière aussi appuyée que les deux barres verticales. Ce chiffre est suivi de la lettre $\mathrm{A}$. Si cette lecture est avérée, $L O$ pourrait alors être l'abréviation de $L O C O$, et il s'agirait des restes d'une marque de carrière pour indiquer l'enregistrement ou le contrôle d'un bloc extrait ${ }^{10}$. Ces types de marques ne sont pas rares sur des blocs de marbre de Carrare ${ }^{11}$, mais peuvent se retrouver aussi sur d'autres pierres ${ }^{12}$. Normalement, ces marques étaient gravées sur les côtés les moins visibles des

\footnotetext{
5. BenoIT 1954, pl. 32, 2.

6. CaIn 1985.

7. CAIN 1985, p. 27-38, annexe 2 .

8. Comme par ex. la base de Palestrina : CaIN 1985, p. 27 $\mathrm{n}^{\circ} 5$; 168 Kat. $\mathrm{N}^{\circ}$ 58, pl. 9, 1-3.

9. Cette lecture m'a été proposée par Patrizio Pensabene que je remercie vivement ici.
}

10. Ch. Dubois, Étude sur l'administration et l'exploitation des carrières : marbres, porphyre, granit, etc. : thèse pour le doctorat, Paris, éd. A. Fontemoing, 1908, p. 84.

11. Pensabene 2013, p. 181-182, fig. 3.13 (bloc de marbre de l'épave de Beauséjour), p. 424.

12. Par ex. sur un granit local à Brage : Pensabene 2013, p. 482 , fig. 12.9 . 


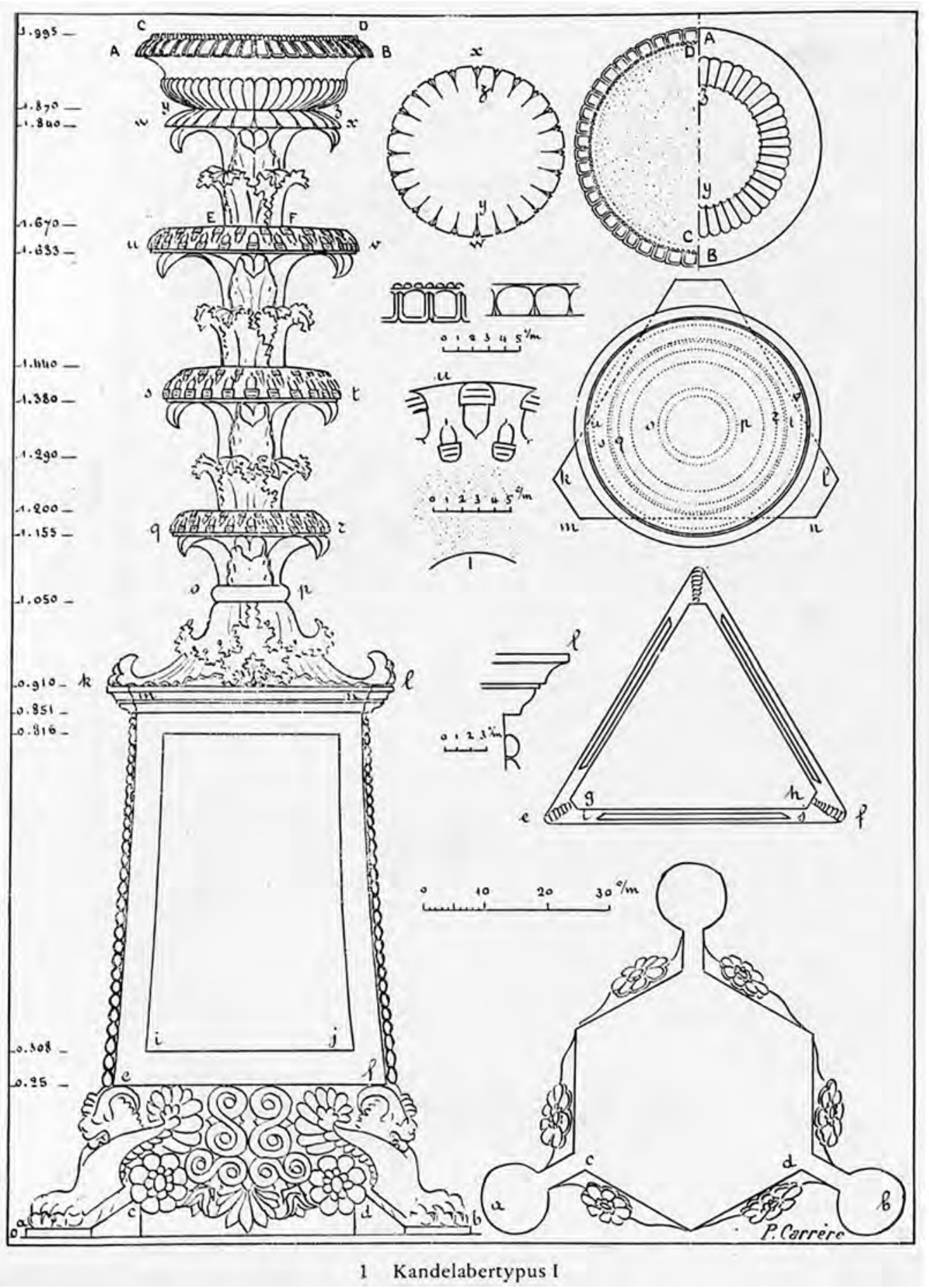

10. Premier type des candélabres brûle-parfum. D'après Cain 1985, annexe 2. 
blocs, ou elles étaient effacées par la suite. La présence de ce graffito en plein champ du relief figuré indique peut-être que le bloc était placé assez haut dans l'édifice, à un niveau de visibilité réduite.

Quant aux quatre figures féminines qui sont presque totalement arasées, elles nous livrent également quelques indices. La draperie flottante derrière le corps de la dernière figure à droite, la position gracieuse des pieds de la première, quelques autres détails des vêtements rappellent des figures dansantes de l'époque hellénistique et leurs reprises par des ateliers néo-attiques. L'antécédent classique du $\mathrm{IV}^{\mathrm{e}}$ s. av. J.-C. qui revient à l'esprit est le monument dit «tribune d'Echmoun " à Sidon ${ }^{13}$ et, surtout, sa frise inférieure qui représente une danse des Nymphes.

Identifier ces figures n'est pas pour autant chose aisée, et pas seulement à cause de leur extrême détérioration. En effet, plusieurs divinités féminines peuvent être représentées en procession, ou en dansant. Les Nymphes, vieilles divinités rustiques, mais également les Charites ou Grâces, les Horai, protectrices de la végétation, représentées en groupe de jeunes filles dansantes, peuvent également être d'éventuelles candidates pour notre relief. Parmi l'abondante iconographie de ces sujets, citons quelques exemples. Sur un autel circulaire en marbre d'époque augustéenne ${ }^{14}$, les Nymphes qui dansent guidées par Pan (fig. 11a-b) peuvent se comparer à nos figures. La figure interprétée comme une des Horai sur une plaque en marbre du Musée national d'Athènes ${ }^{15}$ en est également très proche. Enfin, les scènes de procession ou de farandole, menée par des Charites, comportent des figures tout à fait comparables ${ }^{16}$. Le fait que celles de notre bloc soient drapées les rapproche des prototypes grecs, copiés fréquemment à l'époque romaine, alors que les Grâces romaines sont représentées nues. Nous osons ainsi proposer une restitution hypothétique du décor $\mathrm{du}$ bloc, toutefois incomplète (fig. 12). Pour le détail de la mise en scène nous devons rester prudent, car le fait que la partie supérieure de la frise manque ne permet pas de savoir si les personnages se tenaient par la main, par un bout de leur draperie en exécutant une farandole, ou s'ils portaient des offrandes ou des attributs, comme on le voit sur de nombreux documents.

Préciser le type de monument où pouvait être intégré ce bloc lors de sa première phase est tout aussi difficile. Son appartenance au décor d'un temple semble impossible à cause du fait que la frise se développait sur deux assises. À titre d'hypothèse, on peut penser que ce bloc a pu appartenir au décor d'un monument dédié aux Nymphes, ou d'un autel monumental. En tout cas la présence du brûle-parfum nous place dans l'ambiance d'une cérémonie religieuse luxueuse ${ }^{17}$.

ORIGINE DU BLOC

Les remplois de blocs architecturaux en marbre à Arles sont expliqués, de manière arbitraire, comme des remplois locaux. Cette cuve aurait donc été produite par un atelier local à Arles.

13. R. STUCKY, Tribune d'Echmoun. Ein griechischer Reliefzyklus des IV. Fahrhunderts v. Chr. in Sidon (AntK, Suppl. 13), Bâle, 1984.

14. Vatican, Museo Gregoriano, Prof. 9940 : LIMC, VIII, 1997, s.v. "Nymphai», p. $894 \mathrm{n}^{\circ} 32$ (M. Halm-Tisserant, G. Siebert).
15. Inv. 260 : LIMC, V, 1990, s.v. "Horai", p. $504 \mathrm{n}^{\circ} 10$, pl. 345 (V. Machaira).

16. LIMC, III, 1986, s.v. "Charites", p. $196 \mathrm{n}^{\circ}$ 23-25: copies néo-attiques, datées de l'époque d'Hadrien (E. Harrison).

17. TESCRA, 5, 2005, s.v. "Rauchopfer", p. 212-229 (T. Hölscher). 


\section{- (c) PUF .}
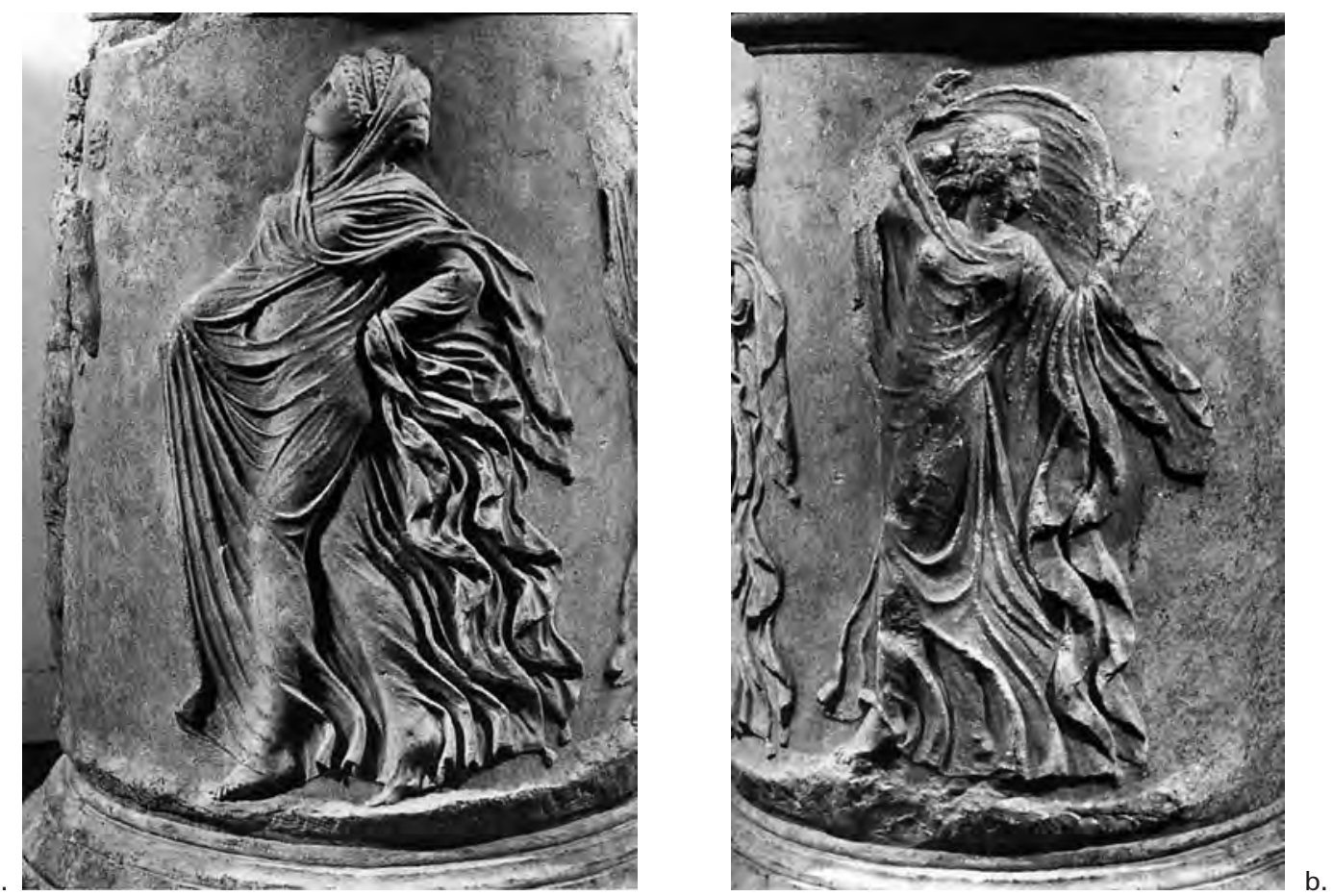

11a-b. Pan et les Nymphes. D'après LIMC, VIII, 1997, s.v. « Nymphai », pl. 589 nº 32.
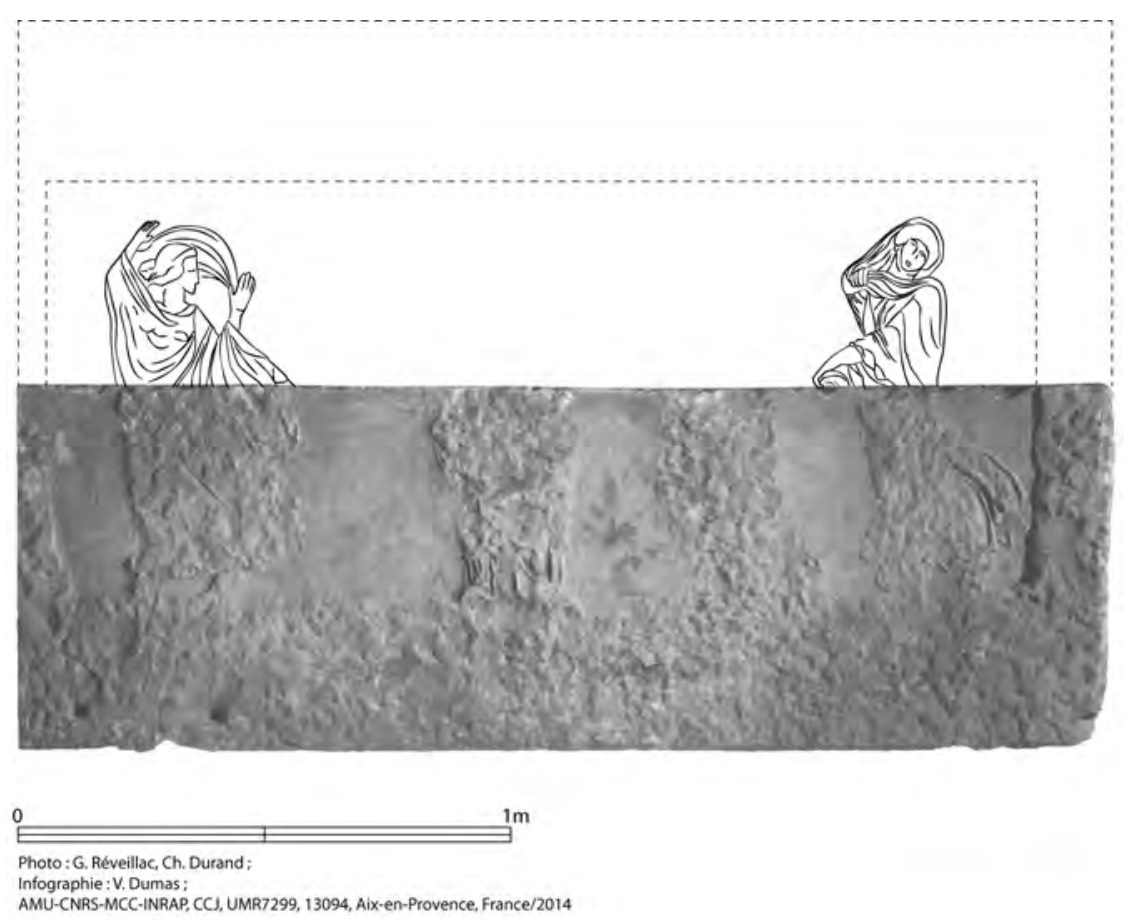

12. Restitution hypothétique du décor du bloc.

(c) Cl. Ch. Durand, G. Réveillac ; infographie V. Dumas ; CNRS, CCJ (2014). 
Pourtant, des cuves découvertes à Rome offrent des parallèles tout à fait convaincants, notamment pour ce qui concerne les nœuds formés par l'étoffe ${ }^{18}$ (fig. 13) et la préciosité des panneaux des strigiles.

Ce sarcophage n'est pas le seul parmi ceux d'Arles à être taillé dans un bloc de remploi, comme je l'ai montré ailleurs ${ }^{19}$. Dans tous les cas il s'agit de blocs de marbre et ce n'est pas pour autant, à mon avis, qu'il faut y voir l'œuvre d'un atelier local qui recyclait des blocs architecturaux. Ce recyclage du matériau a pu se faire à Rome, où ces blocs n'offraient pas seulement le choix d'un moindre coût. À mes yeux, ces techniques sont des témoins bien plus précieux du mode de gestion de la matière première dont disposait un atelier de sculpture qui réunissait un certain nombre d'artisans, comme cela devait être possible dans un atelier de taille importante situé à Rome. Selon Patrizio Pensabene «le centre de Rome était devenu un véritable dépôt de matériel où l'on pouvait s'approvisionner pour

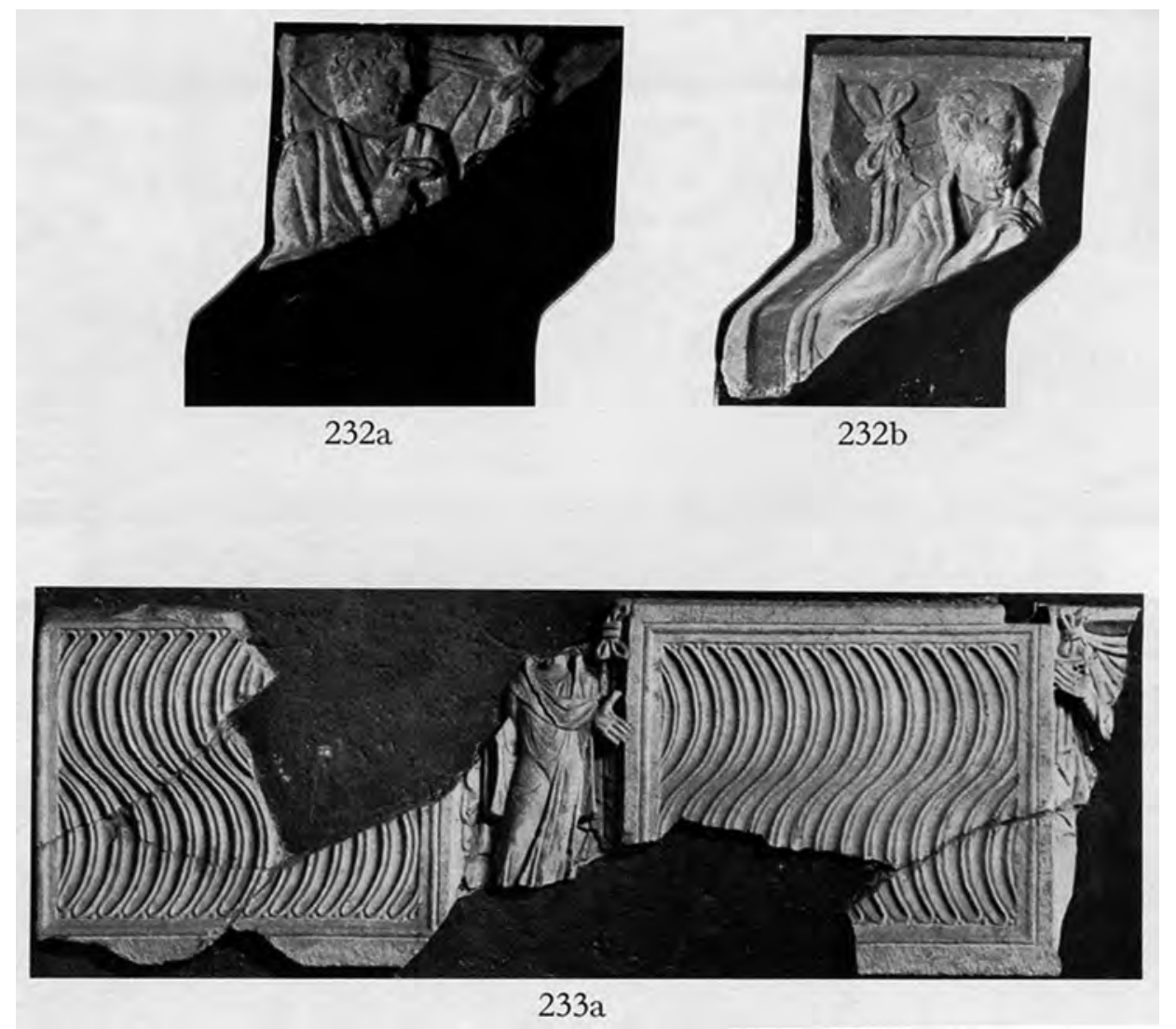

13. Détail d’un sarcophage de Rome. D’après Deichmann, Bovini, Brandenburg 1967, pl. 52 nº $232-233$.

18. Voir trois ex. au Vatican, Museo Pio Cristiano : DeICHMANN, Bovini, BRANDENBuRg 1967, p. 65-67 n 73, 76, 77, pl. 22-23, ou encore deux au cimetière San Sebastiano:
Deichmann, Bovini, Brandenburg 1967, p. 136 n 232-233, pl. 52 .

19. Gaggadis-Robin, Maniatis, Polykreti 2009. 
de nouvelles constructions $»^{20}$, ce qui explique le phénomène du remploi grâce au dépôt de marbres à Rome et à Ostie dans la seconde moitié du $\mathrm{IV}^{\mathrm{e}}$ et au $\mathrm{V}^{\mathrm{e}} \mathrm{s}$., à la suite de la démolition systématique d'édifices publics païens. Bon nombre de ces blocs de corniches, frises etc., se prêtaient par leurs dimensions à la fabrication d'un sarcophage. Les ateliers de production de sarcophages centralisés à Rome avaient certainement accès à ces blocs disponibles. Comme nous venons de le dire, ces remplois se pratiquaient bien à Rome, et l'on aurait tort de les considérer comme ayant été effectués à Arles, ce qui amènerait à penser que soit ces blocs arrivaient prêts au remploi en Arles, soit qu'ils appartenaient à des édifices arlésiens démantelés et remployés sur place. De plus, comme nous venons de le voir, l'exécution de leur décor permet aisément de les rapprocher des productions de Rome.

La prise en compte de ces aspects techniques renforce l'hypothèse que ces pièces ont été fabriquées dans un atelier de Rome qui avait accès aux blocs de marbre à " recycler " dans la capitale. Étant donné l'origine italienne du marbre, le style néo-attique du relief ${ }^{21}$ et le type du brûleparfum représenté, il est fort probable que le bloc ornait à l'origine un monument païen d'époque augustéenne, peut-être à Rome même, et, une fois le monument démantelé, il a été remployé par des ateliers sculptant des sarcophages chrétiens à Rome et importé ensuite à Arles.

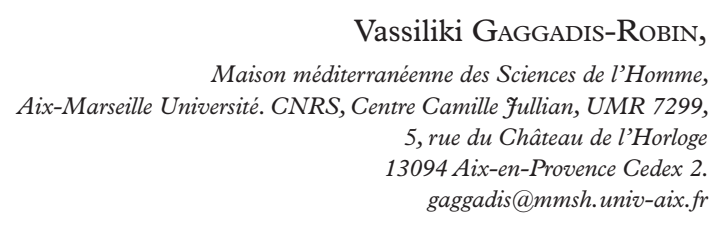

\section{ABRÉVIATIONS BIBLIOGRAPHIQUES}

BENOIT 1954

CAIN 1985

CHRISTERN-BRIESENICK 2003

Deichmann, Bovini,

BRANDENBURG 1967

GAGGADIS-RoBIN 2005

Gaggadis-Robin, Maniatis, Polykreti 2009

Maniatis, DotsiKa, KAVOUSSANAKI 2005

Pensabene 2013 TESCRA
F. Benoit, Les sarcophages paléochrétiens d'Arles et de Marseille (Gallia, Suppl. 5), Paris, CNRS.

H.-U. CAIN, Römische Marmorkandelaber, Mayence, Ph. von Zabern.

B. Christern-BriesENICK, Repertorium der christlich-antiken Sarkophage, III. Frankreich, Algerien, Tunesien, Mayence, Ph. von Zabern.

F. W. Deichmann, G. Bovini, H. Brandenburg, Repertorium der christlichantiken Sarkophage I. Rom und Ostia, Wiesbaden, Franz Steiner.

V. Gaggadis-Robin, Les sarcophages païens du Musée de l'Arles et de la Provence antiques, Arles, Éd. du MAPA.

V. Gaggadis-Robin, Y. Maniatis, K. Polykreti, "Usage du marbre et techniques de fabrication des sarcophages d'Arles ", Ph. Jockey (éd.), Leukos Lithos. Marbres et autres roches de la Méditerranée antique : études interdisciplinaires. Actes duVIII Colloque international de l'Association for the Study of Marble and Other Stones used in Antiquity (ASMOSIA), Aix-en-Provence, 2006, Paris, éd. Karthala, p. 663-667.

Y. Maniatis, E. Dotsika, D. Kavoussanaki, "Techniques utilisées pour déterminer la provenance des quelques sarcophages en marbre du Musée de l'Arles et de la Provence antiques ", GaGgadis-Robin 2005, p. 281-286.

P. Pensabene, I marmi nella Roma antica, Rome, Carrocci.

Thesaurus Cultus et Rituum Antiquorum, Bâle, Union académique internationale.
20. P. Pensabene, «Reimpiego e depositi di marmi a Roma e Ostia tra la seconda metà del IV e i primi decenni del V secolo", M. Fixot (dir.), Paul-Albert Février, de l'Antiquité au Moyen Âge, Actes du colloque de
Fréjus, 7 et 8 avril 2001, Aix-en-Provence, PUP, 2004, p. 281-297.

21. W. Fuchs, Die Vorbilder der neuattischen Reliefs (FdI, Suppl. 20), Berlin, De Gruyter, 1959. 